\title{
Understanding the biologic effect of stress on the developing brain, and insights from the COVID-19 pandemic
}

\author{
Ita Litmanovitz, MD
}

Neonatal Department, Meir Medical Center, Kfar-Saba, Israel, Affiliated to Sackler school of Medicine, Tel-Aviv University, Tel-Aviv, Israel

Target article: Nist MD, Harrison TM, Steward DK (2019). The biological embedding of neonatal stress exposure: A conceptual model describing the mechanisms of stress-induced neurodevelopmental impairment in preterm infants. Res Nurs Health, 42:61-71. DOI: 10.1002/nur.21923

$\mathrm{T}$ he exposure to cumulative stress during critical neurodevelopmental windows early in life is a major unresolved challenge of modern newborn intensive care. In sharp contrast to the soothing environment of the womb, life-saving care provided in the Newborn Intensive Care Unit (NICU) exposes preterm infants to numerous painful and stressful interventions while separated from their mother.

The experience of repetitive exposure to stress places preterm infants at risk since significant maturational processes take place in the fetal brain during the second half of gestation. These include synapse formation (synaptogenesis), programmed cell death (apoptosis), proliferation of glia cells, and the beginning of myelination. As a result of premature birth, these processes occur in a time span when the preterm infant is hospitalized in the NICU. The protection afforded by the intra-uterine environment is no longer available, leaving the infant's developing brain vulnerable to different environmental stressors.

Studies that explored the impact of increased exposure to stress factors in the NICU, ${ }^{1,2}$ demonstrated alterations in brain neural connectivity, increased apoptotic processes that led to decreased regional brain volume and delayed maturation of the white matter at term age. Decreased regional brain volumes were found in former preterm infants even at seven years of age. ${ }^{3}$ Furthermore, a growing body of evidence, from both animal and human studies, indicate that stress experienced during the fetal and neonatal period is associated with substantial long-term neurodevelopmental morbidity. ${ }^{4}$

Despite the increasing evidence regarding the impact of stress on brain development of preterm infants, the mechanisms underlying these short- and long-term developmental influences remain largely unexplored. In the reviewed article, Nist and her colleagues present a new conceptual framework: The Neonatal Stress Embedding (NSE) model. They put forward an explanation grounded on the biological effects that newborn stress exposure in the NICU might have on short- and long-term neurodevelopment. The authors hypothesize that stress affects brain structure and function through alterations in four biological systems: the immune system, the autonomic nervous system
(ANS), the Hypothalamic-Pituitary Axis (HPA), and gene expression. These four systems interact with each other and most importantly, they can be modulated by both pre-natal and postnatal environmental variables like parental stress and maternal interaction (Figure 1).

The NSE conceptual model might be appealing to clinicians since it is consistent with known concepts of the developmental origins of health and illness. The Biological Embedding of Childhood Adversity Model ${ }^{5}$ postulates that early life stress such as childhood maltreatment, neglect, and violence, affects subsequent adult health outcomes. It provided the theoretical framework for the NSE model presented by Nist and colleagues in this review.

Fetal and/or neonatal inflammatory processes have been long associated with adverse neurodevelopmental outcomes in the perinatal literature. ${ }^{6}$ Inflammation is actually considered a common underlying mechanism in the multifactorial origins of several morbidities related to prematurity, such as bronchopulmonary dysplasia (BPD), retinopathy of prematurity (ROP) and necrotizing enterocolitis (NEC). Studies from both adult and neonatal animal models have revealed that chronic stress responses are associated with systemic inflammation. Newborn animal models further suggest that exposure to stress directly activates nervous system cells called microglia, known to play a role as primary regulators of immune responses in the brain. However, studies assessing the effect of stress on the immune function in preterm infants are still missing.

The involvement of both the autonomic nervous system (ANS), and the Hypothalamic-Pituitary Axis (HPA) in stress responses is well documented. Their integration into the NSE model therefore seems logical, yet not thoroughly studied in this age group. In newborn infants, exposure to stress results in increased sympathetic and decreased parasympathetic activity, as measured by changes in heart rate variability (HRV). Limited evidence from infants affected with sepsis suggests that decreased parasympathetic activity measured by HRV is a predictor for future neurologic impairments in preterm infants. However, the change in HRV might only be a marker for central nervous 
FIGURE 1. Neonatal Stress Embedding Model. ANS, autonomic nervous system; HPA, hypothalamic-pituitary-adrenal. (Adapted from Nist et al. 2019).

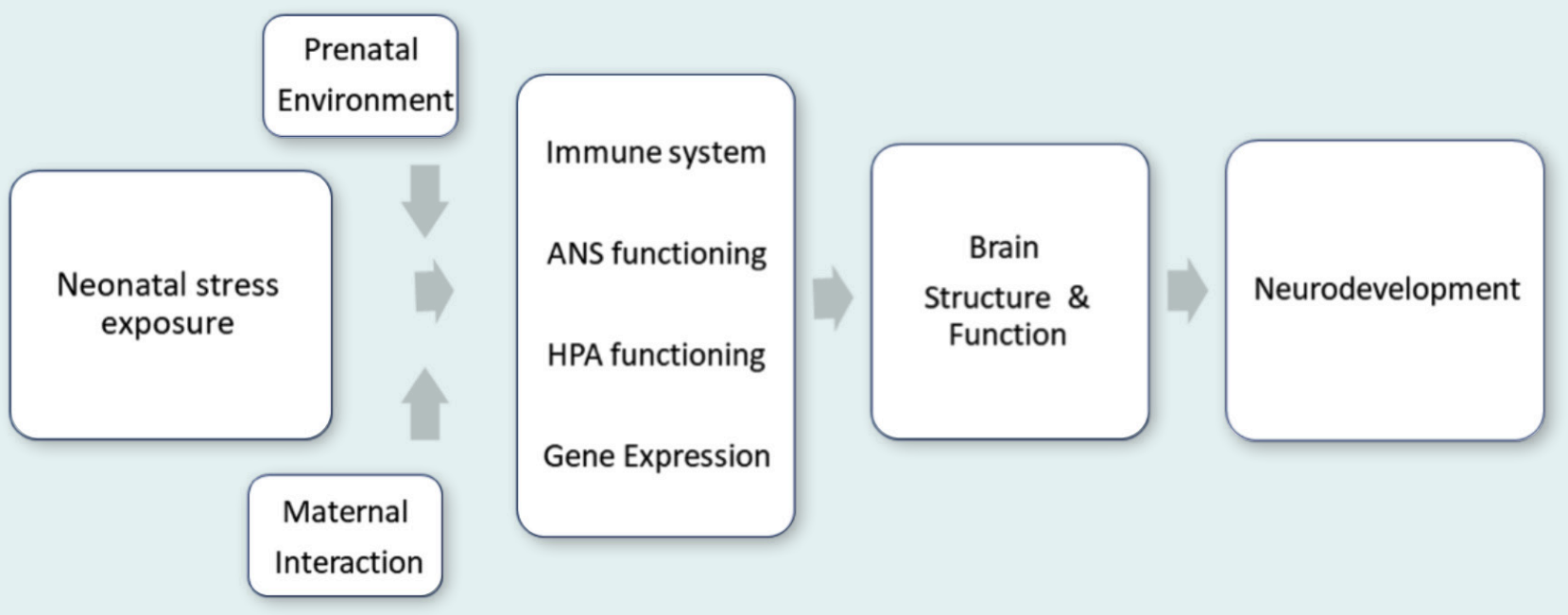

system involvement, rather than the actual cause of the neurologic insult. Chronic stress exposure is also known to cause repeated activation of the HPA axis, resulting in glucocorticoid resistance. Salivary cortisol levels measured before and following exposure to a stressor were lower in preterm as compared to term-born infants. Changes in the HPA response were demonstrated at school age and were associated with cognitive and attention problems.

Many of the mediators linking stress exposure and neurodevelopment are, in turn, mediated by epigenetic changes in gene expression occurring postnatally, as discussed in detail by Jeff Alberts in a recent issue of the Developmental Observer. ${ }^{8}$

The article by Nist and colleagues provides a comprehensive review on the topic of newborn stress and its impact on preterm infants. The article utilizes accepted models of the developmental origins of health and illness to shed new light on the impact of stress on preterm infant brain development. Thereby, it underlines the need for implementing caregiving approaches aimed at reducing and modulating infants' exposure to stressful stimuli and experiences at the bedside - as postulated by NIDCAP-based care - to improve the outcomes of preterm infants.

The limitation of the model is that the evidence base of the proposed concept is not strong enough, as it consists mostly of studies of human adults and of animal studies. However, gaps in evidence surely provide opportunities for new areas of research. Studies that will explore and test the suggested model might help to identify infants at risk as well as interventions needed based on their risk profiles.

The authors emphasize the role of nurses in practice changes (perhaps because the paper is published in a nursing journal) however, optimization of neurodevelopmental outcomes should be the priority for all clinicians caring for preterm infants. Therefore, the model presented might be meaningful for all health caregivers in the NICU - including decision and policy makers -when adopting practices that reduce stress in the NICU.

Reviewing this article in the midst of the COVID-19 pandemic presented a welcome opportunity to reflect on this topic. It assisted me, as a neonatologist, to better cope with the challenges generated for hospitalized infants, families, and health care professionals. During the last months, the lived experience of a global pandemic has been extremely stressful for parents, families, and healthcare professionals; stress that might adversely affect the outcome of the preterm infants.

Within these extreme circumstances, in order to keep infants and health care providers safe, some NICUs have adopted policies that drastically separate these medically fragile infants from their parents. It appears that some of the new guidelines implemented as a result of the pandemic lack a comprehensive perspective, and seem to disregard the basic understanding that parental physical and emotional closeness in early life is a cornerstone of optimal infant growth and development. Maternal stress and depression have been shown to have adverse neurodevelopmental effects in infants ${ }^{9}$ and may enhance the deleterious effect of newborn stress. Conversely, maternal closeness and early interaction may moderate infants' physiologic stress responses, affecting the degree to which stress exposure might impact neurodevelopment. Maternal-infant contact as provided during Kangaroo care can lessen stress responses and promote positive neurodevelopment. ${ }^{10,11}$ More parental presence and holding in the NICU have been found to be associated with better outcomes. ${ }^{12}$ Especially during this stressful time, strategies to enhance sensitive parenting and positive family processes will provide a developmentally appropriate environment. ${ }^{13}$

I started my review by stating that infant exposure to cumulative stress in the NICU is a major unsolved challenge in 
newborn care. When contemplating the NSE model, I deduced the reader might initially be inclined to think mostly about the experience of stress in the NICU and its potential adverse effects on preterm infants' development. This is, in my view, the desired starting point from which to approach the newborn's bedside: to have an awareness of the potential harm that our caregiving and procedures can cause. And yet, when I read the article again and let my background as a NIDCAP-trained and experienced neonatologist guide my reflection, I found myself thinking less about the potentially negative experience of stress, and much more engaged in envisioning the nearly boundless possibilities we have to ease and buffer that stress in the NICU.

Because stress experiences have a biological embedding, as the NSE model proposes, the infant's expectation for maternal closeness, physical contact and relationship is certainly biologically embedded as well. We know the brain of the human infant is wired for relationships and early physical contact. ${ }^{14,15}$ By capitalizing on resources readily available in every NICU (infants, parents, love, and the unspoken yearning for closeness and relationship), we can do a meaningful job in reducing and buffering the experience of stress for preterm infants. A NIDCAP-based education gives us, as clinicians, two unique tools to accomplish this important "stress-reducing" job: our skills to observe infant stress and communicate it to those who care for them, and our unequivocal understanding that parents are the infants' most consistent and reliable caregivers.

\section{References}

1. Smith GC, Gutovich J, Smyser C, Pineda R, Newnham C, Tjoeng TH, Inder T. (2011). Neonatal intensive care unit stress is associated with brain development in preterm infants. Annals of Neurology, 70(4):541-549. https://doi.org/10.1002/ana.22545
2. Vinall J, Miller SP, Bjornson, BH, Fitzpatrick KP, Poskitt KJ, Brant R, Grunau RE. (2014). Invasive procedures in preterm children: Brain and cognitive development at school age. Pediatrics, 133(3):412-421. https://doi.org/10.1542/peds.2013-1863

3. Ranger M, Chau CM, Garg A, Woodward TS, Beg MF, Bjornson B, Grunau RE. (2013). Neonatal pain-related stress predicts cortical thickness at age 7 years in children born very preterm. PLoSONE, 8(10): e76702. https://doi.org/10.371/journal.pone.0076702

4. Cong X, Wu J, Vittner D, Xu W, Hussain N, Galvin S, Henderson WA. (2017). The impact of cumulative pain/stress on neurobehavioral development of preterm infants in the NICU. Early Human Development, 108:9 -16. https://doi.org/10.1016/j.earlhumdev.2017.03.003

5. Berens AE, Jensen SKG, Nelson CA. (2017). Biological embedding of childhood adversity: from physiological mechanisms to clinical implications. BMC Med, 20;15(1):135. doi: 10.1186/s12916-017-0895-4. Review.

6. Carlo WA, McDonald SA, Tyson JE, et al. (2011). Cytokines and Neurodevelopmental Outcomes in Extremely Low Birth Weight Infants. J Pediatr. 159(6): 919-925. .e3. doi: 10.1016/j.jpeds.2011.05.042. PMID:21798559

7. Grunau RE, Cepeda IL, Chau CM, Brummelte S. Weinberg J, Lavoie PM, Turvey SE. (2013). Neonatal pain-related stress and NFKBIA genotype are associated with altered cortisol levels in preterm boys at school age. PLoS ONE, 8(9), e73926. https://doi. org/10.1371/journal. pone.0073926

8. Alberts J. (2020). A Funny Thing Happened on the Way to the Hospital. Developmental Observer, 13 (1):22. DOI: 10.14434/do. v13i1.29094.

9. Stanley C, Murray L, Stein A. (2004). The effect of postnatal depression on mother infant interaction, infant response to the Still-Face perturbation, and the performance on an Instrumental Learning task. Development and Psychopathology, 16:1-18.

10. Feldman R. (2004). Mother-infant skin-to-skin contact and the development of emotion regulation. In S. P. Shohov (Ed.), Advances in psychology research (pp. 113-131). Hauppauge, NY; Nova Science.

11. Feldman R, Eidelman AI, Sirota L, Weller A. (2002). Comparison of skin-to-skin (Kangaroo) and traditional care: parenting outcomes and preterm infant development. Pediatrics, 110, 16-26.

12. Reynolds LC, Duncan MM, Smith GC, Mathur A, Neil J, Inder T, Pineda RG. (2013). Parental presence and holding in the neonatal intensive care unit and associations with early neurobehavior. Journal of Perinatology 33:636-641.

13. Pineda R, Bender J, Hal IB, Shabosky L, Annecca A, Smith J. (2018). Parent participation in the neonatal intensive care unit: Predictors and relationships to neurobehavior and developmental outcomes. Early Hum Dev,117:32-38. doi: 10.1016/j.earlhumdev.2017. PMID: 29275070

14. Schore AN (1994). Affect Regulation and the Origins of the Self: The Neurobiology of Emotional Development: Hillsdale, NJ

15. Siegel DJ (1999). The developing mind. How relationships and the brain interact to shape who we are. 3rd Ed. The Guilford Press.
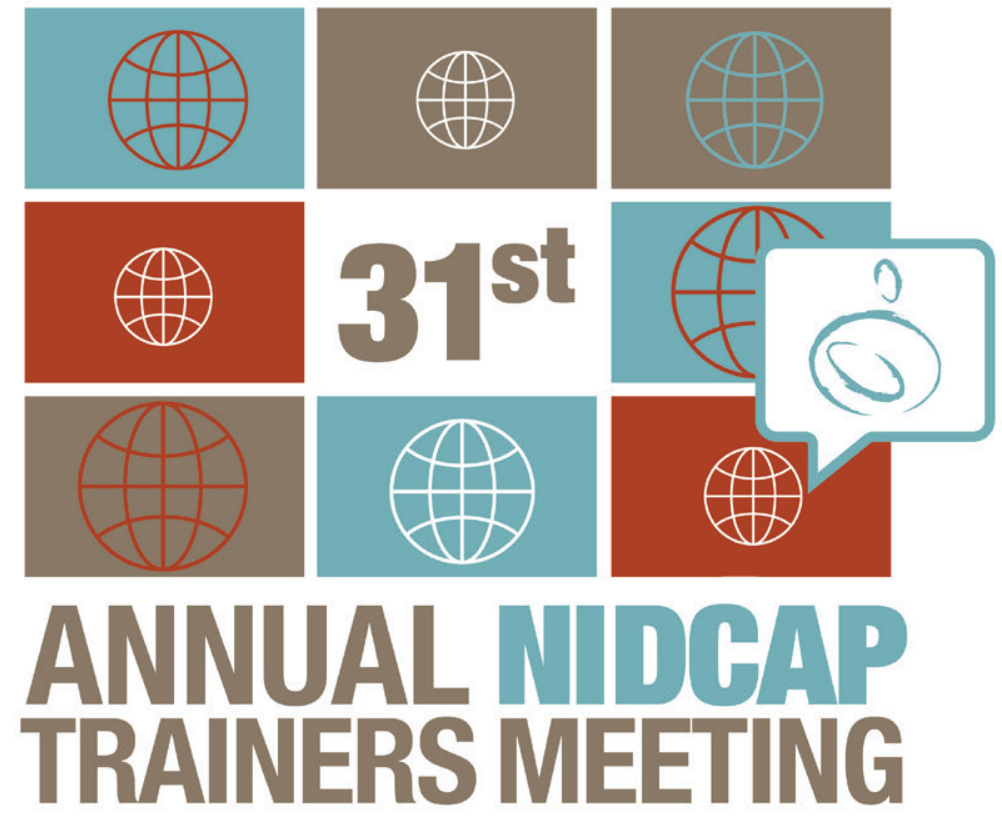

\section{Implementing NIDCAP with New Challenges, New Ways to Connect}

\author{
Hosted by the NFI \\ 21-23 October 2020 \\ 1800 - 2130 GMT
}
This will be a virtual meeting by invitation only, using an online platform. Further details will be circulated via the NFI GoogleGroup list.

